\title{
A New Chemo-Dynamical Tool to Study The Evolution of Galaxies in the Local Universe
}

\author{
Nicolas Champavert, and Hervé Wozniak
}

Centre de Recherche Astronomique de Lyon, France; email: champavert@obs.univ-lyon1.fr

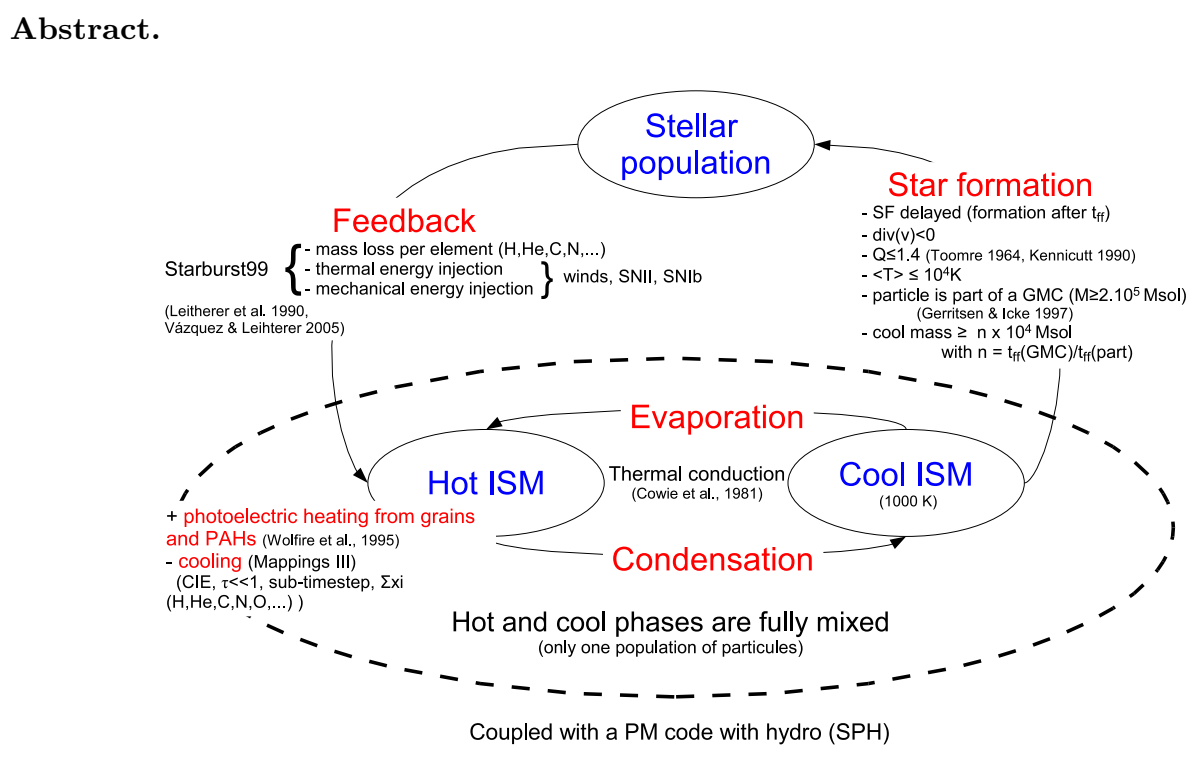

Figure 1. Schematic presentation of our new chemo-dynamical tool. See Champavert \& Wozniak (2007a) for full details and references.

Keywords. galaxies: evolution, ISM: evolution

Fig. 1 displays the main recipes of our new chemodynamical tool developed to describe the evolution of galaxies using a non-instantaneous recycling approach (Champavert \& Wozniak, 2007a). We follow individual abundances for several elements very important for the reconstruction of accurate cooling functions in real time (Champavert \& Wozniak, 2007b). The ISM thermal state and enrichment by SN feedback and stellar winds is thus accurately computed.

Using a toy model, we have shown that compared to another code that only used tabulated cooling curves at fractional solar metallicity, the cooling timescales are longer leading to lower star formation rate.

\section{References}

Champavert, N. \& Wozniak H. 2007a, in: Chemodynamics: from first stars to local galaxies, CRAL Conference Series I, Emsellem et al. (eds), in press.

Champavert, N. \& Wozniak, H. 2007b, in: Heating vs. Cooling in Galaxies and Clusters of Galaxies, ESO Astrophysics Symposia Series, Garching, Boehringer et al. (eds), in press. 